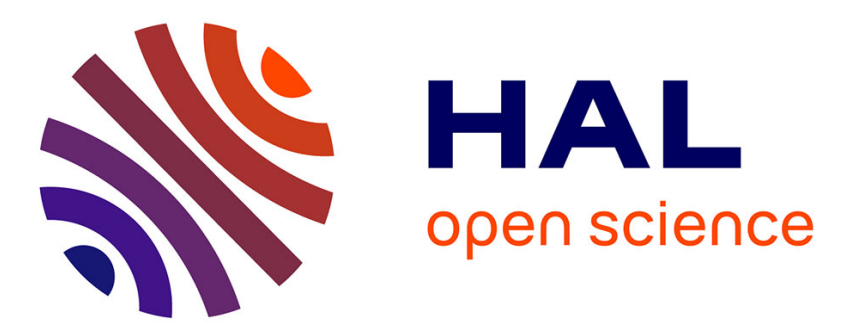

\title{
Dynamical measurement of domain-wall nucleation and annihilation in individual amorphous Co particles
}

W. Wernsdorfer, Klaus Hasselbach, A. Sulpice, J.-E Wegrowe, L. Thomas, B. Barbara, D. Mailly

\section{- To cite this version:}

W. Wernsdorfer, Klaus Hasselbach, A. Sulpice, J.-E Wegrowe, L. Thomas, et al.. Dynamical measurement of domain-wall nucleation and annihilation in individual amorphous Co particles. Physical Review B: Condensed Matter and Materials Physics (1998-2015), 1996, 53 (6), pp.3341. hal-01659954

\section{HAL Id: hal-01659954 https://hal.science/hal-01659954}

Submitted on 9 Dec 2017

HAL is a multi-disciplinary open access archive for the deposit and dissemination of scientific research documents, whether they are published or not. The documents may come from teaching and research institutions in France or abroad, or from public or private research centers.
L'archive ouverte pluridisciplinaire HAL, est destinée au dépôt et à la diffusion de documents scientifiques de niveau recherche, publiés ou non, émanant des établissements d'enseignement et de recherche français ou étrangers, des laboratoires publics ou privés. 


\title{
Dynamical measurement of domain-wall nucleation and annihilation in individual amorphous Co particles
}

\author{
W. Wernsdorfer \\ Centre de Recherches sur les Très Basses Températures, CNRS, Boîte Postale 166, 38042 Grenoble Cedex 9, France \\ and Laboratoire de Magnétisme Louis Néel, CNRS, Boîte Postale 166, 38042 Grenoble Cedex 9, France \\ K. Hasselbach, A. Sulpice, and A. Benoit \\ Centre de Recherches sur les Très Basses Températures, CNRS, Boîte Postale 166, 38042 Grenoble Cedex 9, France \\ J.-E. Wegrowe, L. Thomas, and B. Barbara \\ Laboratoire de Magnétisme Louis Néel, CNRS, Bôte Postale 166, 38042 Grenoble Cedex 9, France \\ D. Mailly \\ Laboratoire de Microstructure et Microéléctronique, CNRS, 196 Avenue Henri Ravera, 92220 Bagneux, France
}

(Received 20 July 1995)

\begin{abstract}
We present magnetization measurements of individual amorphous Co particles $(300 \mathrm{~nm} \times 200 \mathrm{~nm} \times 30 \mathrm{~nm})$ patterned by electron-beam lithography. The hysteresis loops show mainly two magnetization jumps corresponding to domain-wall nucleation and annihilation. The angular dependence of these magnetization jumps is measured and the temperature dependence $(0.1-6 \mathrm{~K})$ of the nucleation and the annihilation processes is investigated by two independent methods: switching field and switching time measurements. Finally we compare the results obtained on one particle with those of an array of about $2 \times 10^{7}$ particles. We show experimentally that the dynamical properties measured on individual particles are connected with an unusual $1 / T$ relaxation of the array of particles.
\end{abstract}

\section{INTRODUCTION}

Since the pioneering work of Néel, ${ }^{1}$ the underlying mechanisms of magnetization reversal in a single magnetic particle have been much discussed. Knowledge of those processes is very basic in magnetism and is based on the StonerWohlfarth model or numerical micromagnetic calculations. A good understanding of the general problem of magnetization reversal in complex systems such as assemblies of particles, thin films, bulk materials, etc., requires knowledge of magnetization reversal processes in a single magnetic particle. These processes have essential implications in recording media applications. The rapidly increasing density of magnetic storage poses the question of how far the reduction of volume-to-surface ratio can continue without inducing new sources of information errors. Until recently all studies were limited to samples consisting of billions of presumably identical particles. Most of the single-particle properties were hidden behind some distribution functions of particle size, shape, etc. Furthermore, particle-particle interactions are difficult to take into account. With the recent arrival of nearfield microscopy and nanolithography, experimental studies $^{2-5}$ of magnetization reversal in individual particles become possible.

In this paper we report on studies of the dynamics and temperature dependence of magnetization reversal in individual submicronic Co particles at very low temperatures. First, we describe briefly our measuring device and the samples. Then we characterize our samples magnetically in measuring the angular dependence of the coercive field. We present two independent techniques to access the dynamics of magnetization switching in a single particle. Finally, we present the relaxation measurements of an array of about $2 \times 10^{7}$ Co particles and compare the obtained results to those of a single particle.

\section{METHOD}

Our approach consists in the use of a planar microbridge superconducting quantum interference device (SQUID) (of 1 $\mu \mathrm{m}$ diameter) on which we place one ferromagnetic particle (see Fig. 1). The SQUID loop collects the flux produced by the sample's magnetization. Due to the close proximity between sample and SQUID, we have a very efficient and direct flux coupling. The SQUID's are made of $\mathrm{Nb}$ and are operational at temperatures as high as $7 \mathrm{~K}$. To prevent trapped flux in the SQUID, the SQUID is made by only one thin $\mathrm{Nb}$ layer (thickness $20 \mathrm{~nm}$ ). Today, in this configuration,

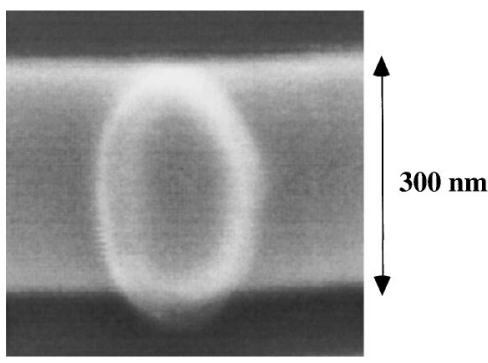

FIG. 1. Electron micrograph showing the wire of a microbridge SQUID with a Co particle (ellipticity $300 \mathrm{~nm} \times 200 \mathrm{~nm}$, thickness $30 \mathrm{~nm}$ ) (white spot) which is placed on the SQUID wire. 


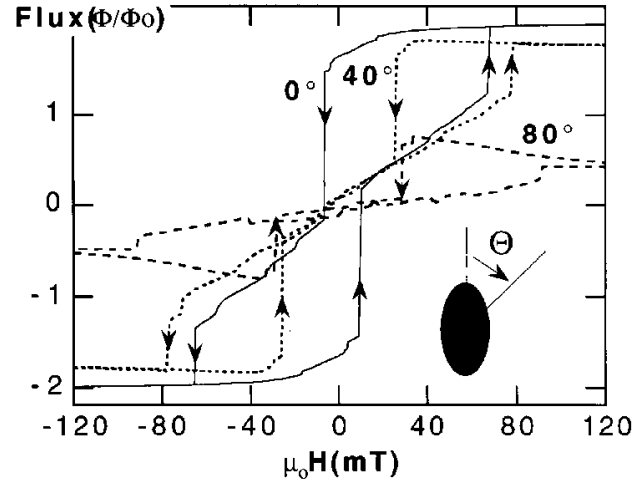

FIG. 2. Hysteresis loops of the Co particle (Fig. 1) for three different angles between the easy axis of magnetization and the in-plane applied field. The detected flux change is proportional to the magnetization only for small angles. Due to a geometrical effect of flux linkage, the hysteresis loop is deformed at higher angles.

we can detect magnetization reversals corresponding to $10^{4}$ $\mu_{B} \cdot{ }^{4}$ The applied field is generated by a solenoid and two pairs of Helmholtz coils. The field can be oriented anywhere in space; its maximal amplitude is $0.5 \mathrm{~T}$. The angle resolution of the applied field is given by the smallest field step of $7 \times 10^{-6}$ T, i.e., much smaller than $0.1^{\circ}$. The first alignment between the long axis of the particle and the applied field is better than $5^{\circ}$. Sweeping rates between 0.01 and $100 \mathrm{mT} / \mathrm{s}$ are used. The field noise is smaller than $10^{-6} \mathrm{~T}$ at $0.5 \mathrm{~T}$.

\section{SAMPLES}

In this paper we focus on 30-nm-thick elliptic Co particles defined by liftoff techniques out of sputtered thin films (protected from oxidation by a $10 \mathrm{~nm}$ thin $\mathrm{Si}$ film). Prior to nanofabrication, we checked the properties of our Co films. $\mathrm{X}$-ray diffraction evidenced a nanocrystalline structure $(5-10 \mathrm{~nm})$. Microprobe analysis revealed small traces of $\mathrm{C}$ and $\mathrm{Ar}$, and a coercive field value of $3 \mathrm{mT}$ at $4 \mathrm{~K}$ was measured by vibrating sample magnetometry. Therefore we conclude that crystalline anisotropy can be neglected and the main anisotropy of our particles is shape anisotropy.

The nanofabricated particles have an elliptic contour with in-plane dimensions of $300 \mathrm{~nm} \times 200 \mathrm{~nm}$ and a thickness of $30 \mathrm{~nm}$ (Fig. 1). We verified the particle's shape by scanning electron microscopy. We also fabricated a sample consisting of $1.8 \times 10^{7}$ identical Co particles (same fabrication method as the individual particle). These particles are placed on a $\mathrm{Si}$ substrate with a spacing of $2 \mu \mathrm{m}$. Because of this large spacing, dipole-dipole interactions between the particles are negligible.

\section{DOMAIN-WALL STRUCTURE AND ANGULAR DEPENDENCE OF DOMAIN-WALL NUCLEATION AND ANNIHILATION}

In order to study the domain structure of our particles, we measure the angular dependence of hysteresis loops. Figure 2 shows typical hysteresis loops of an individual elliptic Co particle as shown in the micrograph of Fig. 1. The magnetic field is applied in the plane of the particle and the SQUID, and the angle $(\theta)$ is measured between the ellipse's long axis

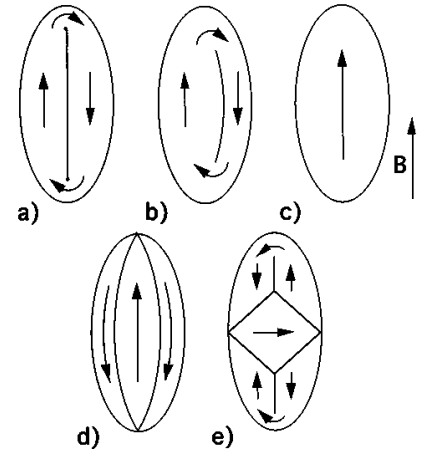

FIG. 3. Schematic drawing of the domain-wall structure in a elliptical particle as proposed by van den Berg. Arrows indicate the spin direction. (a) and (b) show the one-domain wall structure. The field is applied along the long axis as indicated: (a) $B=0$, (b) $B$ is smaller than the saturation field, and (c) $B$ is higher than the saturation field. (d) and (e) show examples of composite structures at $B=0$.

and the applied field. These hysteresis loops are mainly characterized by two magnetization jumps. Starting from the saturation state, the first jump can be associated with domain-wall nucleation and the second jump with domainwall annihilation. During these jumps, the magnetization switches in less than $100 \mu \mathrm{s}$ (our time resolution). The reversible central region of the hysteresis loops is evidence for the motion of the domain wall through the particle. As the detected flux change is proportional to the magnetization only for small angles, the hysteresis loop is deformed at higher angles. This is a pure geometric effect of flux linkage. For example, in the limit of strong fields the captured flux decreases continuously as the angle is increased.

The simplest domain structure, showing such a hysteresis loop, has been proposed by van den Berg ${ }^{6}$ in zero field and calculated for fields smaller than the saturation field by Bryant and Suhl. ${ }^{7}$ This domain structure has been observed experimentally on low-anisotropy circular thin film disks (100 $\mu \mathrm{m}$ diameter) using high-resolution Kerr techniques. ${ }^{8}$ Similar measurements were performed on rectangular Permalloy particles, for example, by Hefferman et al. ${ }^{9}$ In zero field, the particle is divided in two equal domains by a single $180^{\circ}$-domain wall, as shown in Fig. 3(a). When a magnetic field is applied, this domain wall is pushed to the order of the particle [Fig. 3(b)]. For higher fields the domain wall is annihilated and the particle becomes single domain [Fig. 3(c)]. The main conditions for this model are (i) that the magnetic material is very soft and (ii) that the system is two dimensional. The first condition is satisfied by our particles as they are made of a randomly oriented nanocrystallized Co, being very soft. The second condition is also quite well satisfied as the demagnetizing field perpendicular to the plane is much larger than the in-plane demagnetizing field. Furthermore, we obtained similar results for thinner particles (10 or 20 $\mathrm{nm})$. More complicated domain structures [e.g., Figs. 3(d) and 3(e)] may be excluded by the fact that similar Co particles of length smaller than $200 \mathrm{~nm}$ are single domain., ${ }^{4,5}$

In Fig. 4 we show the angular dependence of the switching fields corresponding to domain-wall nucleation and annihilation for the Co particle of Fig. 1. In the following, as the symmetry of the data is about fourfold, we limit our 


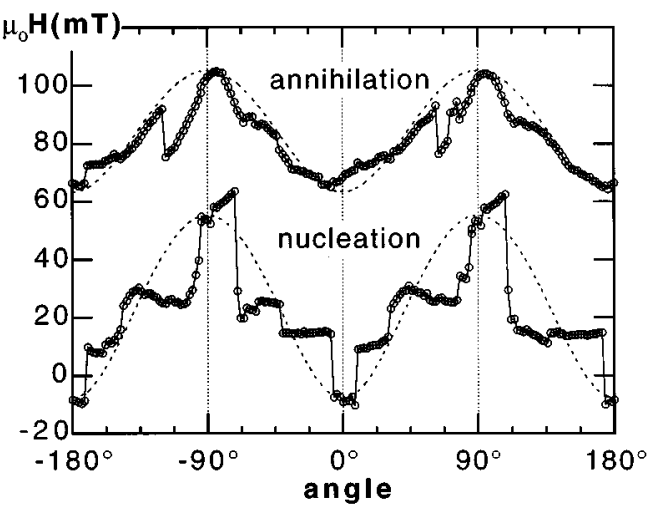

FIG. 4. Angular dependence of the switching fields for the Co particle shown in electron micrograph of Fig. 1. The lower switching field corresponds to domain-wall nucleation, whereas the higher one corresponds to domain-wall annihilation. The data are fitted by a simple model described in the text (dotted lines).

description to the interval $0^{\circ}<\theta<90^{\circ}$. The domain-wall nucleation field is negative near $0^{\circ}$ and increases for higher angles. This angle dependence is not continuous, being probably due to surface defects. The domain-wall annihilation field is also increasing for higher angles. This can be explained by the following picture. At high fields the particle's magnetization is saturated. When the field is reduced, the demagnetizing field of the particle tends to nucleate a domain wall to reduce the magnetostatic energy. In order to nucleate this domain wall, the sum of the demagnetizing and applied fields must be greater than the nucleation field. Once the domain wall is nucleated, the domain wall moves towards an equilibrium position where the sum of the demagnetization and applied fields is zero. In increasingly larger fields, the domain wall is pushed near the border of the particle. When the distance between the border of the particle and the center of the domain wall is of the order of the domain-wall thickness, the domain wall annihilates. As the magnetostatic energy of the particle is an increasing function of the angle, the domain wall nucleates already at higher fields as the field is lowered. Similarly higher fields must be applied to annihilate the domain wall. For example, the magnetostatic energy of a single domain particle increases for higher angles as $E=-K \cos ^{2}(\psi)$, where $K$ is the energy of anisotropy [e.g., for shape anisotropy $K$ $=\mu_{0} M_{s}^{2}\left(N_{y}-N_{x}\right) / 2$ (Ref. 10), where $N_{x}$ for the long axis and $N_{y}$ for the short axes are the in-plane demagnetizing factors] and $\psi$ is the angle between the $x$ axis and the magnetization of the particle.

The general variation of Fig. 4 can be recovered very roughly if we assume that the measured nucleation field is given by

$$
H_{N \text { measured }}=H_{D}(\theta)-H_{N},
$$

where $H_{D}$ is the angular dependence of the demagnetizing field and $H_{N}$ is the nucleation field (we do not account for the angular dependence of $H_{N}$ and the magnetization is supposed to be parallel to the applied field, $\psi=\theta$ ). By using the expression $H_{D}^{2}=M_{S}^{2}\left(N_{X}^{2} \cos ^{2} \theta+N_{Y}^{2} \sin ^{2} \theta\right),{ }^{10}$ Eq. (1) becomes

$$
H_{N \text { measured }}=M_{S} \sqrt{N_{Y}^{2}+\left(N_{X}^{2}-N_{Y}^{2}\right) \cos ^{2} \theta}-H_{N} .
$$

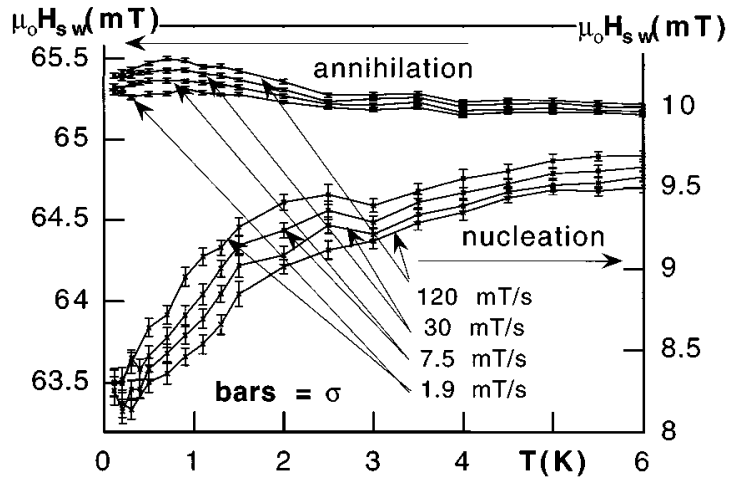

FIG. 5. Variation of the mean switching fields, corresponding to domain-wall nucleation and annihilation, of the Co particle (photo 1) as a function of temperature and for four field sweeping rates. The width $\sigma$ of the switching field distributions are indicated by vertical bars.

The nucleation field at $\theta=0$ corresponds to $H_{N}$ measured $(0)$ $=M_{S} N_{X}-H_{N}$ and at $\theta=\pi / 2$ it is $H_{N \text { measured }}(\pi / 2)$ $=M_{S} N_{Y}-H_{N}$. The difference $H_{N \text { measured }}(\pi / 2)$ $-H_{N \text { measured }}(0)=M_{S}\left(N_{Y}-N_{X}\right)$ is the shape anisotropy field which is related to the shape anisotropy $K$ $=\mu_{0} M_{s}^{2}\left(N_{y}-N_{x}\right) / 2$.

The calculation of the annihilation field is similar, but we have to invert one sign in Eq. (1): $H_{A \text { measured }}=H_{D}(\theta)$ $+H_{A}$.

Thus we can explain why we observe the $180^{\circ}$ periodicity in contrast to the $90^{\circ}$ periodicity of the Stoner-Wohlfarth model and why the fields are maximum for applied field directions perpendicular to the easy axis.

We applied this simple model on the measurements of the nucleation and annihilation fields (dotted lines in Fig. 4). Using the bulk magnetization of saturation of Co $\left(M_{S}=1.4 \times 10^{6} \mathrm{~A} / \mathrm{m}\right.$ and the thickness $(30 \mathrm{~nm})$ and length $(300 \mathrm{~nm})$ of the particle, we found for the domain-wall nucleation $\mu_{0} H_{N}=(138 \pm 12) \mathrm{mT}$ and for the width of the particle, $b=(230 \pm 6) \mathrm{nm}$. For the domain-wall annihilation, we found $\mu_{0} H_{A}=(68 \pm 7) \mathrm{mT}$ and $b=(252 \pm 4) \mathrm{nm}$. These results can be compared with the real width of the particle of $b=200 \mathrm{~nm}$. The fact that the anisotropy field of the particle, $\mu_{0} H_{K}=\mu_{0} M_{s}\left(N_{y}-N_{x}\right)=92 \mathrm{mT}$, is much larger than the nucleation field (see Fig. 5), suggests that the particle undergoes incoherent reversal.

In the following, the field is always applied almost parallel to the long axes of the particle (at $10^{\circ}$ in Fig. 4).

\section{DYNAMICS OF DOMAIN-WALL NUCLEATION AND ANNIHILATION}

After having characterized the static magnetic properties, we use two independent techniques to access to the dynamical reversal properties. We will call the first approach switching field measurements and the second switching time measurements. In the first case, the applied field is changed at a given rate and fixed temperature and the field value is stored as soon as the sample magnetization switches. After about 100 cycles, switching field histograms are established, allowing us to define mean nucleation and annihilation fields and 

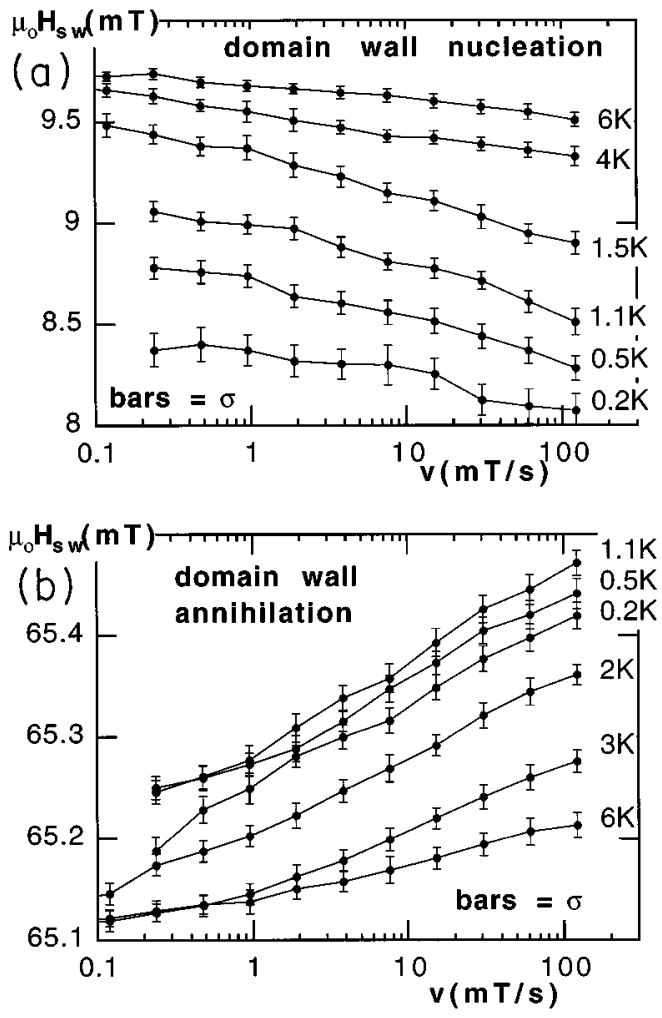

FIG. 6. The influence of the sweeping rate on the mean switching field corresponding to (a) domain-wall nucleation and (b) domain-wall annihilation for six temperatures. The width $\sigma$ of the switching field distribution is indicated by vertical bars.

their respective (rms deviation) $\sigma$ of the switching distributions. In contrast to this, for switching time experiments, we increase at a given temperature the magnetic field until the set point is reached. Then we measure the time it takes until magnetization switches; this process is repeated again about 100 times, and we obtain switching time histograms of the nucleation and the annihilation process. Integration of these histograms gives the probabilities of nucleation and annihilation.

\section{A. Switching field measurements}

At each temperature the switching fields are obtained for field sweeping rates between 0.01 and $100 \mathrm{mT} / \mathrm{s}$. As expected for thermal activated switching, the mean nucleation field increases with increasing temperature $(0.1-6 \mathrm{~K})$ (i.e., the nucleation takes place earlier at higher temperatures), whereas the annihilation field decreases (Fig. 5) as the domain wall moves more easily through defects. However, for temperatures below $1.5 \mathrm{~K}$, the temperature variation of the annihilation field flattens and then decreases below $1 \mathrm{~K}$. Figures 6(a) and 6(b) show the sweeping rate dependence of the mean nucleation and annihilation switching fields. Again, as expected for thermally activated switching, the switching field decreases with increasing sweeping rate $v$ in the case of the nucleation process and increases in the case of the annihilation process. A simple fit of the experimental data can be obtained by $H_{\mathrm{SW}}=a+b \ln (v)$, where the constants $a$ and $b$ are dependent on temperature. Surprisingly, the constant $b$ increases for decreasing temperature. Furthermore, the width

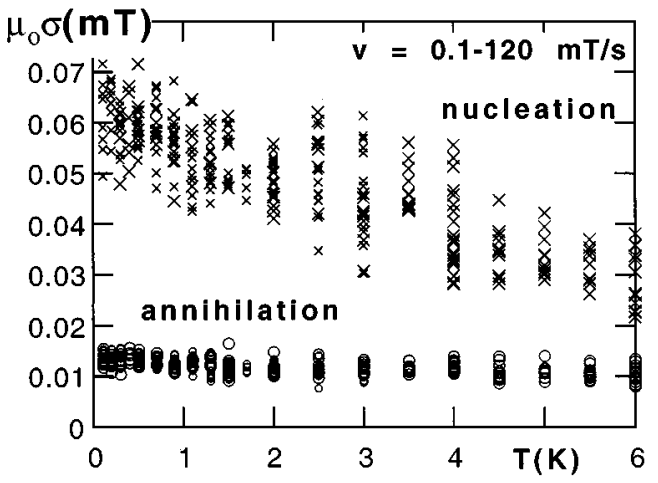

FIG. 7. Temperature dependence of the widths of the switching field distributions $\sigma$ for the Co particle.

$\sigma$ of the switching field distributions (given by the bars in Figs. 5 and 6 and plotted in Fig. 7) increases for decreasing temperature. A possible explication is given below. Finally, $\sigma$ decreases with sweeping rate, but this dependence is very weak.

\section{B. Switching time measurements}

Via the switching time measurements, we have direct access to the switching probability. Figures $8(a)-8(d)$ show examples of the measured probability of switching of domain-wall nucleation and annihilation for 0.1 and $6 \mathrm{~K}$. To compare the measurements with a model of a single energy barrier, we have fitted the data by a stretched exponential

$$
P(t)=e^{-(t / \tau)^{\beta}},
$$

where $\tau$ defines the mean waiting time and $\beta$ gives the deviation of the system from the model of a single energy barrier $(\beta=1)$. The field dependence of $\tau$ is well fitted by the empirical law $\tau(T, H)=\tau_{0} c(T) \exp \left[ \pm H / H_{0}(T)\right]$, where the positive constants $c$ and $H_{0}$ depend on the temperature. The plus sign is for the nucleation process and the minus sign for annihilation process. Figure 9(a) shows the field dependence of $\tau$ for the domain-wall nucleation process. The temperature dependence of $H_{0}$ for the nucleation and annihilation processes is presented in Fig. 9(b). $H_{0}$ decreases for increasing temperature, which is different from an Arrhenius law where $H_{0}$ would be proportional to the temperature. Figure 10 shows the temperature dependence of $\beta$ (at $\tau=10 \mathrm{~s}$ ). For the domain-wall nucleation process, $\beta$ is about 1 , whereas for the domain-wall annihilation process, $\beta$ is about 2 . In both cases, $\beta$ decreases for temperature below $1 \mathrm{~K}$.

\section{ARRAY OF Co PARTICLES}

After studying the dynamical magnetization reversal of individual particles, the question arises of which manner the properties of a macroscopic sample are based on one-particle properties. To answer this question, we fabricated a sample consisting of $1.8 \times 10^{7}$ identical elliptic Co particles of about the same dimensions and material as the individual particle studied above. These particles are placed on a $\mathrm{Si}$ substrate with a spacing of $2 \mu \mathrm{m}$. Because of this large spacing, dipole interactions between particles are negligible. Figure 11 shows the hysteresis loop of the array of Co particles when 

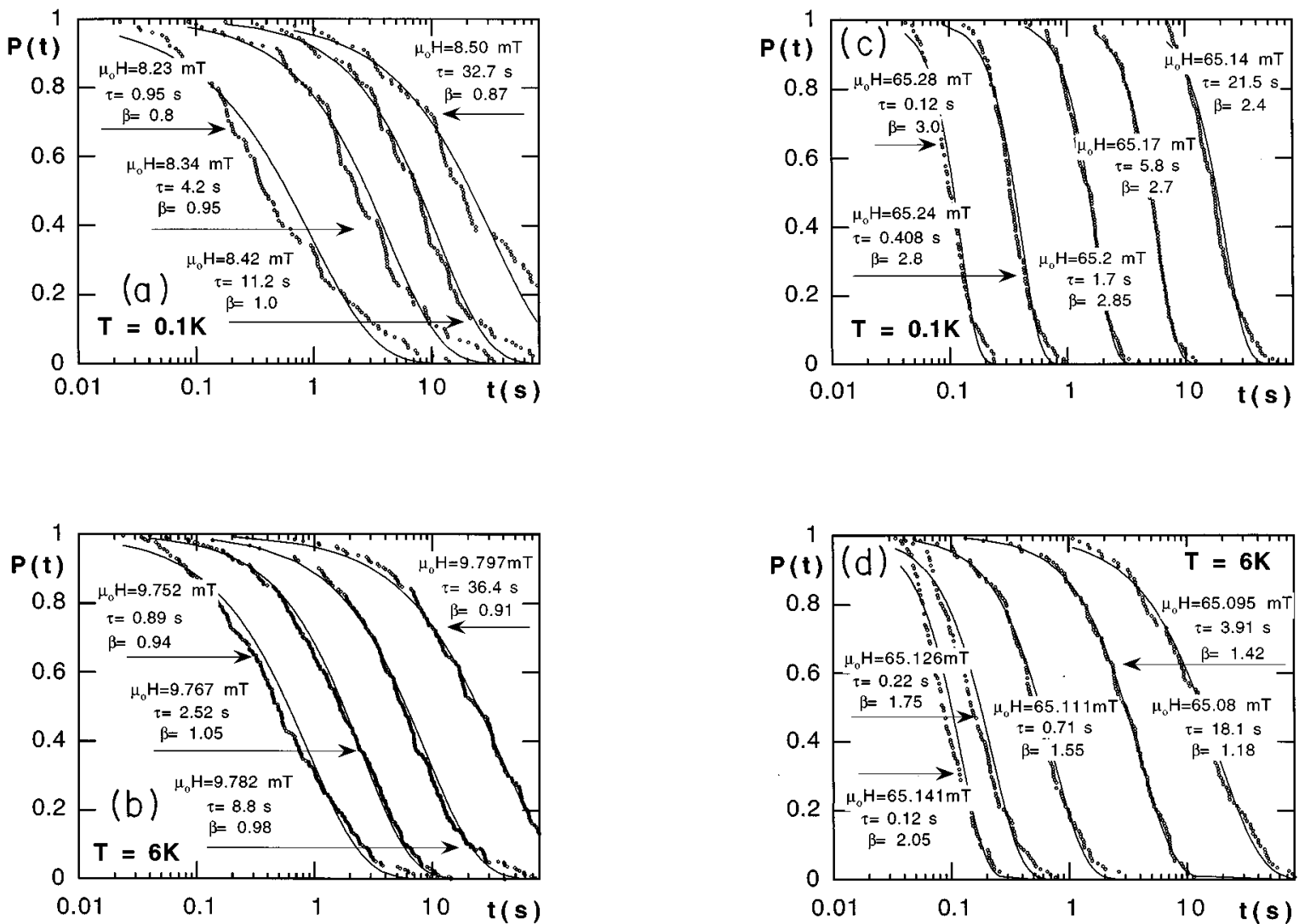

FIG. 8. Probability of not switching of magnetization as a function of the time at different applied fields for domain-wall nucleation at (a) $0.1 \mathrm{~K}$ and (b) $6 \mathrm{~K}$ and for domain-wall annihilation at (c) $0.1 \mathrm{~K}$ and (d) $6 \mathrm{~K}$. Solid lines are fits to the data with a stretched exponential as given by formula (3). The fitting parameters $\beta$ and $\tau$ are indicated in the graphs. The mean-square errors $\chi^{2}$ for the fits in (a) $-(\mathrm{d})$ are between 0.0002 and 0.005 .

the field is applied parallel to the long axis of the particle. This hysteresis loop shows the same characteristics as the hysteresis lop of one particle (Fig. 2), i.e., nucleation and annihilation of domain walls. Because of switching field distributions mainly due to surface defects and a slight distribution of particle sizes, shapes, etc., the domain-wall nucleation and annihilation are no longer discontinuous, but take place along a continuous curve with a width of about $10 \mathrm{mT}$.

Magnetization relaxation experiments of the array (these measurements are equivalent measurement to the switching time measurements for one particle) were performed using a commercial SQUID magnetometer [Metronique Instruments, sensitivity better than $\left.10^{-10} \mathrm{~A} \mathrm{~m}^{2}\left(10^{-7} \mathrm{emu}\right)\right]$. In the following, we consider only the nucleation process of the domain wall. First, we apply a magnetic field of $150 \mathrm{mT}$ to saturate the sample (initial magnetic state). After $15 \mathrm{~min}$, the field is decreased within $1-2$ min to the desired relaxation field $(5 \mathrm{mT}<H<25 \mathrm{mT})$. Then, the relaxation of the magnetization was measured during $3 \mathrm{~h}$. We found a logarithmic relaxation in the time interval of $10^{2} \mathrm{~s}<t<10^{4} \mathrm{~s}$ as seen in Fig. 12. From these measurements, it is possible to obtain the magnetic viscosity, defined as $S=d M(t) / d[\ln (t)]$. Figure 13 shows the relaxation field dependence of $S$ for different temperatures. The data can be well fitted by a Gaussian line shape, and the coercive field can be defined by the maximum of this curve. The width of the Gaussian is between $7.5 \mathrm{mT}$ at $2 \mathrm{~K}$ and $5 \mathrm{mT}$ at $10 \mathrm{~K}$. The temperature dependence of the maximum is less than $0.5 \mathrm{mT}$ between 2 and $10 \mathrm{~K}$, which is quite similar to the individual particle (Fig. 5). Common models of magnetization relaxation show that, at low temperature, the magnetic viscosity is nearly proportional to the temperature. ${ }^{11}$ In our case we found an unusual $1 / T$ temperature dependence of $S$ (Fig. 14). For temperatures higher than $10 \mathrm{~K}$, the magnetization relaxation is smaller than the sensitivity of the magnetometer $\left(10^{-10} \mathrm{~A} \mathrm{~m}^{2}\right)$.

\section{DISCUSSION}

We propose that the magnetization reversal of our particles is governed by two mechanisms. The first one comes from sample defects (surface roughness, crystalline defects, impurities, etc.), creating a multivalleyed energy landscape. These defects are less important at high temperatures when the thermal energy $(k T)$ is much larger than the energy landscape fluctuations due to the defects. At lower temperatures, when $k T$ becomes of the order of the energy fluctuations or smaller, the magnetization reversal becomes sensitive to these fluctuations. As a consequence, the system can have different ways to overcome the energy barrier which may differ slightly from one cycle to another. During the hysteresis loop, the system chooses randomly a path through the energy landscape. It moves along a valley lowering its energy, but it cannot change its valley as the thermal energy is not sufficient to jump over saddle points. As the thermal 

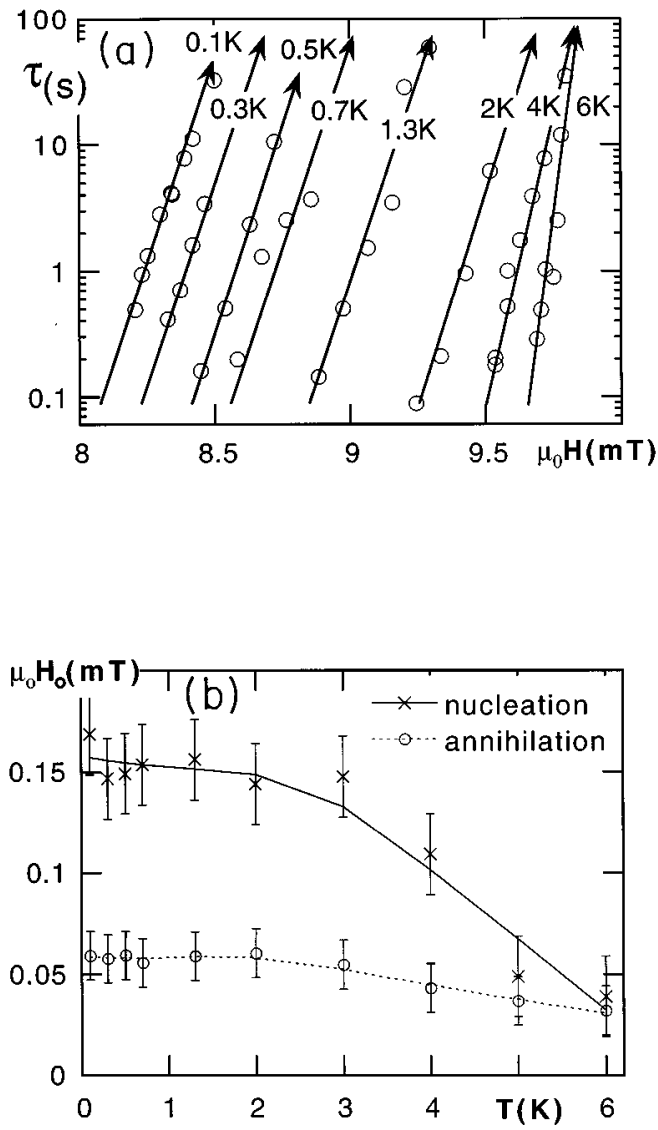

FIG. 9. (a) Field dependences of the mean switching time $\tau$ at different temperatures for domain-wall nucleation. The data are fitted to the empirical law $\tau(T, H)=\tau_{0} c(T) \exp \left( \pm H / H_{0}(T)\right)$. (b) The temperature dependence of $H_{0}$ for the nucleation and annihilation process. For an Arrhenius law, $H_{0}$ would be proportional to the temperature.

energy lowers, this effect will become more and more important and may be at the origin of the increasing width of the switching field distribution (Fig. 7). Furthermore, this mechanism may be responsible for the decrease of $\beta$ with temperature $(\beta<1)$. It is interesting to note that the width of the switching field distribution of domain-wall nucleation is much larger than the width of domain-wall annihilation. The reason may be due to the fact that there are more defects on

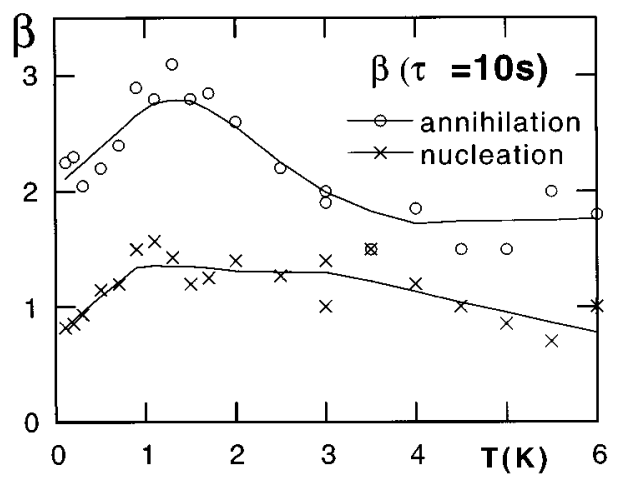

FIG. 10. Temperature dependence of the stretching exponents $\beta$ (for $\tau=10 \mathrm{~s}$ ) as used for fitting the probabilities of not switching [Eq. (3)].

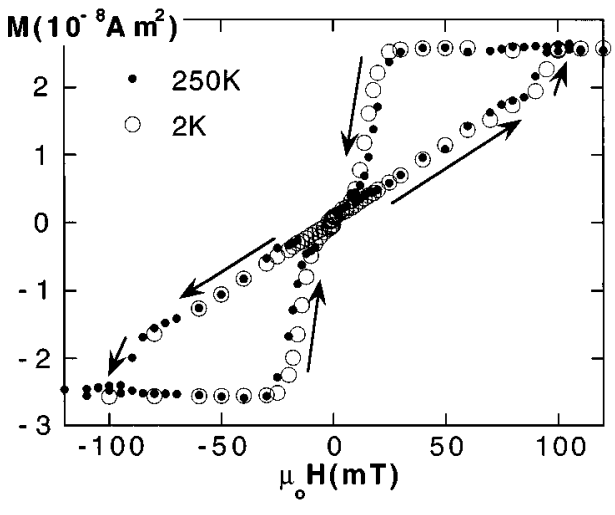

FIG. 11. Hysteresis loops of the magnetic moment of the array of $1.8 \times 10^{7}$ Co particles $(300 \mathrm{~nm} \times 200 \mathrm{~nm} \times 30 \mathrm{~nm})$ for 2 and 250 $\mathrm{K}$. The in-plane field is applied along the long axis of the particles.

the surface governing the domain-wall nucleation than defects in the particle influencing the domain-wall annihilation.

The second mechanism governing the magnetization reversal of our particles is due to the fact that these particles are relatively large compared with the exchange correlation length. Therefore, at the edge of a particle, the moments are not collinear with the bulk magnetization and, on lowering the field, the magnetization reverses via fanning and/or vortex formation of the moments. ${ }^{12}$ These formations may not be stable near the switching fields and may relax slightly, which can be seen as a relaxation of the energy barrier. It is thermally activated; i.e., it is more and more blocked for decreasing temperatures. The relaxation is particularly strong in the case of domain-wall annihilation as the domain wall in the particle involves a lot of spins (the length of the domain wall is of the order of the particle length). This mechanism may be the origin of $\beta>1$, of the decreasing slope $b(T)$ for increasing temperatures [sweeping rate dependence of the mean switching fields, $\left.H_{\mathrm{SW}}=a+b \ln (v)\right]$, and of the decreasing of the constant $H_{0}(T)$ for increasing temperatures (field dependence of the mean switching time, $\left.\tau(T, H)=\tau_{0} c\left\{(T) \exp \left[ \pm H / H_{0}(T)\right]\right\}\right)$. Finally, it may influence in the temperature dependence of the magnetic viscosity of the array of particles. The relaxation of the magnetization at very short times is faster at higher temperatures than

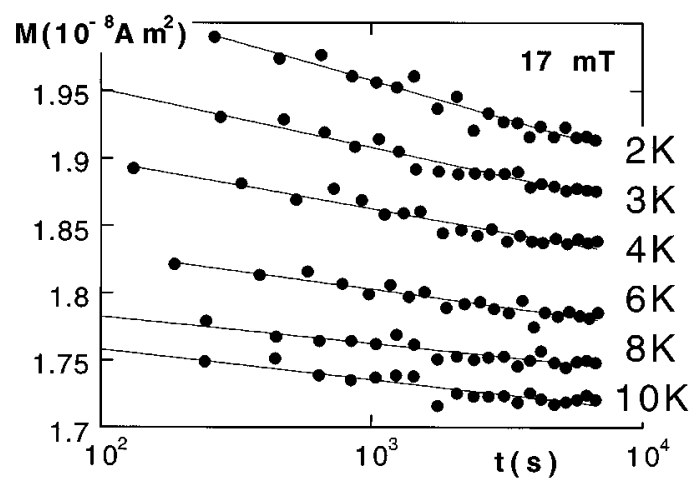

FIG. 12. Relaxation of magnetization of the array of Co particles at $H=17 \mathrm{mT}$. The slope $S=d M / d[\ln (t)]$ defines the magnetic viscosity. 


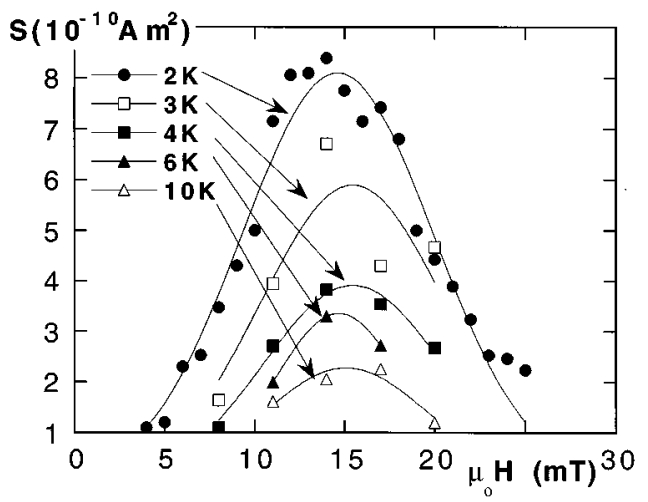

FIG. 13. Field dependence of the magnetic viscosity $S=d M / d[\ln (t)]$ for several temperatures. The data are fitted by Gaussian functions.

at lower temperatures. As a consequence, the relaxation at longer time is slow because there are not many particles left which can still relax. When the temperature is decreased, the relaxation becomes more and more blocked at very short times. As a consequence, the relaxation at longer time can be faster as there are more particles left over. In other words, the fast relaxation of magnetization occurring at the beginning of the experiment is more and more delayed as the temperature is lowered. Therefore the increasing magnetic viscosity at lower temperatures is possible because there are more particles left which nucleate a domain wall in the experimental time interval $\left(10^{2} \mathrm{~s}<t<10^{4} \mathrm{~s}\right)$. The fact of more unswitched particles at lower temperature can also be seen in the inset of Fig. 14 showing the magnetization at $t=10^{4} \mathrm{~s}$ as a function of temperature. The experimental results can also be described in terms of linear response to a small field near the nucleation field. ${ }^{13}$

\section{CONCLUSION}

The main results of this article are the following. The hysteresis loops of amorphous Co particles are characterized by two magnetization jumps being due to domain-wall

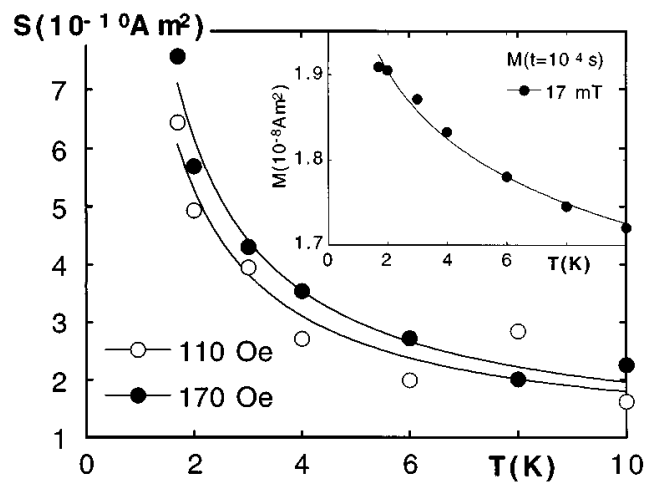

FIG. 14. Temperature dependence of the magnetic viscosity $S=d M / d[\ln (t)]$ at 11 and $17 \mathrm{mT}$. The data are fitted by a $1 / T$ dependence. The inset shows the magnetization at $t=10^{4} \mathrm{~s}$ as a function of temperature.

nucleation and annihilation processes. Dynamical measurements show thermally activated switching fields. Switching time probabilities are faster than exponential. Furthermore, relaxation measurements of an array of Co particles show an unusual $1 / T$ temperature dependence of the magnetic viscosity.

These results can be described by two mechanisms: (i) Particle defects are responsible for a multivalley energy landscape revealed as the thermal energy lowers. This mechanism may be at the origin of the increasing width of the switching field distribution. (ii) As the particles are relatively large, the energy barrier is slightly relaxing when a field is applied which is near the switching field. This mechanism may be responsible for the enhanced exponential switching probabilities $(\beta>1)$.

\section{ACKNOWLEDGMENTS}

We want to acknowledge the important contributions of $\mathbf{J}$. L. Porteseil, B. Pannetier, and T. Crozes to the success of this work. The authors are indebted to GEC ALSTHOM, Belfort, France for providing the superconducting wire.
${ }^{1}$ L. Néel, Ann. Geophys. 5, 99 (1949).

${ }^{2}$ M. Ledermann, S. Schulz, and M. Ozaki, Phys. Rev. Lett. 73, 1986 (1994).

${ }^{3}$ T. Chang and J. G. Chu, J. Appl. Phys. 75, 5553 (1994).

${ }^{4}$ W. Wernsdorfer, K. Hasselbach, D. Mailly, B. Barbara, A. Benoit, L. Thomas, and S. Suran, J. Magn. Magn. Mater. 145, 33 (1995)

${ }^{5}$ W. Wernsdorfer, K. Hasselbach, A. Benoit, G. Cernicchiaro, D. Mailly, B. Barbara, and L. Thomas, J. Magn. Magn. Mater. 151, 38 (1995).

${ }^{6}$ H. A. M. van den Berg, J. Appl. Phys. 61, 4194 (1987).

${ }^{7}$ P. Bryant and H. Suhl, Appl. Phys. Lett. 54, 78 (1989).

${ }^{8}$ M. Rührig, W. Bartsch, M. Vieth, and A. Hubert, IEEE Trans.
MAG-26, 2807 (1990).

${ }^{9}$ S. J. Hefferman, J. N. Chapman, and S. McVitie, J. Magn. Magn. Mater. 95, 76 (1991).

${ }^{10}$ E. C. Stoner and E. P. Wohlfarth, Philos. Trans. London A 240, 599 (1948); L. Néel, C. R. Acad. Sci. 224, 1550 (1947).

${ }^{11}$ R. Street and J. C. Wooley, Proc. Phys. Soc. London A 62, 562 (1949); L. Néel, C. R. Acad. Sci. 228, 664 (1949).

${ }^{12}$ M. E. Schabes and H. N. Bertram, J. Appl. Phys. 64, 1347 (1988); D. R. Fredkin et al., ibid. 69, 5276 (1991); Y. Nakatani, N. Hayashi, and Y. Uesaka, Jpn. J. Appl. Phys. 30, 2503 (1991).

${ }^{13}$ J.-E. Wegrowe, W. Wernsdorfer, L. Thomas, B. Barbara, A. Sulpice, K. Hasselbach, A. Benoit, and D. Mailly, Phys. Rev. B (to be published). 